Supporting Information for

\title{
Kinetic and reactive distillation for acrylic acid synthesis via transesterification
}

\begin{abstract}
CuncunZuo, Tingting Ge, Chunshan Li*, Shasha Cao, Suojiang Zhang*
Beijing Key Laboratory of Ionic Liquids Clean Process, Key Laboratory of Green

Process and Engineering, State Key Laboratory of Multiphase Complex Systems, Institute of Process Engineering, Chinese Academy of Sciences, Beijing 100190, PR
\end{abstract}

\section{China}

To whom correspondence should be addressed.

E-mail: csli@ipe.ac.cn\& sjzhang@ipe.ac.cn

1. Experimental and calculated VLLE data for the acrylic acid/methyl acrylate and acetic acid/methyl acrylate binary systems

The multicomponent expressions for the activity coefficient for the NRTL and UNIQUAC equations are expressed by Eqs (1) and (2).And the experimental and calculated VLLE data for the acrylic acid/methyl acetate and acetic acid/methyl acrylate binary systems are clearly listed in Table S1 and Table S2, respectively.

$$
\begin{gathered}
\ln \gamma_{i}=\frac{\sum_{j=1}^{c} \tau_{j i} G_{j i} x_{j}}{\sum_{k=1}^{c} G_{k i} x_{k}}+\sum_{j=1}^{c} \frac{x_{j} G_{i j}}{\sum_{k=1}^{c} G_{k i} x_{k}}\left[\tau_{i j}-\left(\sum_{r=1}^{c} x_{r} \tau_{r j} G_{r j} / \sum_{k=1} G_{k i} x_{k}\right)\right](1) \\
G_{i j}=\exp \left(-\alpha_{i j} \tau_{i j}\right) ; G_{j i}=\exp \left(-\alpha_{i j} \tau_{j i}\right) \\
\tau_{i j}=a_{i j}+\frac{b_{j i}}{T} ; G_{i i}=1 ; \tau_{i i}=\tau_{j j}=0 ; \alpha_{i j}=\alpha_{j i}
\end{gathered}
$$

where $T$ is the absolute temperature $(\mathrm{K}) ; a_{i j}$ and $b_{j i}$ are the NRTL binary interaction parameters.

$$
\ln \gamma_{i}=\ln \frac{\phi_{i}}{x_{i}}+\frac{z}{2} q_{i} \ln \frac{\theta_{i}}{\phi_{i}}+l_{i}-\frac{\phi_{i}}{x_{i}} \sum_{j=1}^{n c} x_{j} l_{j}+q_{i}^{\prime}\left[1-\ln \left(\sum_{j=1}^{n c} \theta_{j}^{\prime} \tau_{j i}\right)-\sum_{j=1}^{n c} \frac{\theta_{j}^{\prime} \tau_{j i}}{\sum_{k=1}^{n c} \theta_{k}^{\prime} \tau_{j i}}\right]
$$




$$
\begin{gathered}
\phi_{i}=\frac{r_{i} x_{i}}{\sum_{k=1}^{n c} r_{k} x_{k}}, \quad \theta_{i}=\frac{q_{i} x_{i}}{\sum_{k=1}^{n c} q_{k} x_{k}}, \quad \theta_{i}^{\prime}=\frac{q_{i}^{\prime} x_{i}}{\sum_{k=1}^{n c} q_{k}^{\prime} x_{k}} . \\
\tau_{i j}=\exp \left(a_{i j}+\frac{b_{i j}}{T}\right), \quad l_{i}=\frac{z}{2}\left(r_{i}-q_{i}\right)-\left(r_{i}-1\right) \quad \text { and } \mathrm{z}=10 .
\end{gathered}
$$

Where $T$ is the absolute temperature $K$; and $a_{i j}$ and $b_{i j}$ are the UNIQUAC binary interaction parameters.

Table S1. Experimental VLE data and calculated data for acrylic acid(1) and methyl

\begin{tabular}{|c|c|c|c|c|c|c|c|}
\hline \multicolumn{4}{|c|}{$T / \mathrm{K}$} & \multicolumn{4}{|c|}{ VLE } \\
\hline$T^{\operatorname{Exp}}$ & $T^{\text {Est }}{ }_{\text {NRTL }}$ & $T^{\text {Est }}$ UNIQ & $x_{1}^{\text {Exp }}$ & $x_{1}{ }^{\mathrm{Est}-\mathrm{N} / \mathrm{U}}$ & $y_{1}{ }^{\text {Exp }}$ & $y_{1}{ }^{\text {Est-N }}$ & $y_{1}{ }^{\text {Est-U }}$ \\
\hline 329.13 & 330.1099 & 330.1099 & 0 & 0 & 0 & 0 & 0 \\
\hline--- & 330.7003 & 330.5048 & --- & 0.02 & --- & 0.000506 & 0.001035 \\
\hline--- & 331.1035 & 330.6197 & --- & 0.04 & --- & 0.000894 & 0.001248 \\
\hline--- & 331.4379 & 330.7095 & --- & 0.06 & --- & 0.001202 & 0.001359 \\
\hline--- & 331.7414 & 330.8405 & --- & 0.08 & --- & 0.00147 & 0.00149 \\
\hline--- & 332.0417 & 331.0348 & --- & 0.1 & --- & 0.001726 & 0.001661 \\
\hline--- & 332.358 & 331.2982 & --- & 0.12 & --- & 0.001992 & 0.00188 \\
\hline--- & 332.703 & 331.6307 & --- & 0.14 & --- & 0.002282 & 0.002152 \\
\hline 333.16 & 333.0845 & 332.0295 & 0.148 & 0.16 & 0.00105 & 0.002609 & 0.002483 \\
\hline--- & 333.5101 & 332.4923 & --- & 0.18 & --- & 0.002982 & 0.002879 \\
\hline--- & 333.9803 & 333.0157 & --- & 0.2 & --- & 0.003412 & 0.00335 \\
\hline--- & 334.5002 & 333.5996 & --- & 0.22 & --- & 0.00391 & 0.003906 \\
\hline--- & 335.0689 & 334.2395 & --- & 0.24 & --- & 0.004488 & 0.004559 \\
\hline--- & 335.6884 & 334.9318 & --- & 0.26 & --- & 0.005159 & 0.005324 \\
\hline 336.14 & 336.3593 & 335.6823 & 0.27212 & 0.28 & 0.00592 & 0.005938 & 0.006216 \\
\hline--- & 337.0825 & 336.4864 & --- & 0.3 & --- & 0.006842 & 0.007253 \\
\hline--- & 337.8586 & 337.3437 & --- & 0.32 & --- & 0.007891 & 0.008457 \\
\hline--- & 338.6885 & 338.254 & --- & 0.34 & --- & 0.009107 & 0.009851 \\
\hline--- & 339.5685 & 339.2173 & --- & 0.36 & --- & 0.010516 & 0.011463 \\
\hline 340.18 & 340.5101 & 340.2338 & 0.37514 & 0.38 & 0.01138 & 0.012148 & 0.013322 \\
\hline--- & 341.5092 & 341.3041 & --- & 0.4 & --- & 0.014038 & 0.015464 \\
\hline--- & 342.5671 & 342.4287 & --- & 0.42 & --- & 0.016226 & 0.017928 \\
\hline--- & 343.6854 & 343.6085 & --- & 0.44 & --- & 0.018759 & 0.020758 \\
\hline 345.17 & 344.8613 & 344.8444 & 0.46805 & 0.46 & 0.0162 & 0.021685 & 0.024005 \\
\hline--- & 346.1045 & 346.1376 & --- & 0.48 & --- & 0.025071 & 0.027727 \\
\hline--- & 347.4141 & 347.4896 & --- & 0.5 & --- & 0.028985 & 0.031989 \\
\hline
\end{tabular}
acetate(2)using the NRTL and UNIQUAC models. 


\begin{tabular}{cccccccc}
--- & 348.7926 & 348.9021 & --- & 0.52 & --- & 0.033509 & 0.036866 \\
350.19 & 350.2424 & 350.3768 & 0.52001 & 0.54 & 0.0465 & 0.038736 & 0.042444 \\
--- & 351.7663 & 351.9161 & --- & 0.56 & --- & 0.044776 & 0.04882 \\
353.12 & 353.3672 & 353.5225 & 0.56197 & 0.58 & 0.04753 & 0.051752 & 0.056105 \\
--- & 355.0479 & 355.199 & --- & 0.6 & --- & 0.059807 & 0.06443 \\
--- & 356.8118 & 356.949 & --- & 0.62 & --- & 0.069106 & 0.073942 \\
358.17 & 358.6622 & 358.7764 & 0.66399 & 0.64 & 0.08327 & 0.079838 & 0.084811 \\
--- & 360.6029 & 360.6857 & --- & 0.66 & --- & 0.092219 & 0.097237 \\
--- & 362.6375 & 362.6818 & --- & 0.68 & --- & 0.106495 & 0.111447 \\
--- & 364.7703 & 364.7707 & --- & 0.7 & --- & 0.12295 & 0.127708 \\
--- & 367.0058 & 366.959 & --- & 0.72 & --- & 0.141905 & 0.14633 \\
368.19 & 369.349 & 369.254 & 0.72247 & 0.74 & 0.16181 & 0.163726 & 0.167674 \\
--- & 371.8052 & 371.6643 & --- & 0.76 & --- & 0.188826 & 0.192161 \\
--- & 374.3808 & 374.1994 & --- & 0.78 & --- & 0.217677 & 0.220281 \\
--- & 377.0849 & 376.87 & --- & 0.8 & --- & 0.250845 & 0.252603 \\
380.2 & 379.9208 & 379.6882 & 0.8307 & 0.82 & 0.3114 & 0.28885 & 0.28979 \\
--- & 382.901 & 382.6673 & --- & 0.84 & --- & 0.332409 & 0.332606 \\
--- & 386.0373 & 385.8221 & --- & 0.86 & --- & 0.382286 & 0.381923 \\
--- & 389.3441 & 389.1685 & --- & 0.88 & --- & 0.439346 & 0.438728 \\
393.25 & 392.8392 & 392.7238 & 0.89959 & 0.9 & 0.51062 & 0.50457 & 0.504114 \\
--- & 396.5479 & 396.5058 & --- & 0.92 & --- & 0.579055 & 0.579255 \\
--- & 400.4919 & 400.5323 & --- & 0.94 & --- & 0.664182 & 0.665361 \\
--- & 404.7175 & 404.8203 & --- & 0.96 & --- & 0.761399 & 0.763581 \\
--- & 409.2742 & 409.3846 & --- & 0.98 & --- & 0.872575 & 0.874937 \\
414.20 & 414.2326 & 414.2327 & 1.00 & 1 & 1 & 1 & 1 \\
\hline
\end{tabular}

Table S2. Experimental VLE data and calculated data for acetic acid (1) and methyl acrylate (2)using the NRTL and UNIQUAC models.

\begin{tabular}{ccccccccc}
\hline \multicolumn{4}{c}{$T / \mathrm{K}$} & \multicolumn{9}{c}{ VLE } \\
\hline$T^{\text {Exp }}$ & $T^{\text {Est }}$ NRTL & $T^{\text {Est }}$ UNIQ & $x_{1}{ }^{\text {Exp }}$ & $x_{1}{ }^{\text {Est-N/U }}$ & $y_{1}{ }^{\text {Exp }}$ & $y_{1}{ }^{\text {Est-N }}$ & $y_{1}{ }^{\text {Est-U }}$ \\
353.6 & 353.5419 & 353.5419 & 0 & 0 & & 0 & 0 & 0 \\
--- & 354.1484 & 354.1415 & --- & 0.02 & --- & 0.006827 & 0.006737 \\
--- & 354.6097 & 354.5967 & --- & 0.04 & --- & 0.01346 & 0.013093 \\
--- & 355.0206 & 355.0056 & --- & 0.06 & --- & 0.019453 & 0.018827 \\
--- & 355.406 & 355.3937 & --- & 0.08 & --- & 0.024951 & 0.02416 \\
--- & 355.7821 & 355.7763 & --- & 0.1 & --- & 0.030176 & 0.029323 \\
356.17 & 356.16 & 356.1635 & 0.14323 & 0.12 & 0.03775 & 0.035326 & 0.034497 \\
--- & 356.5479 & 356.5618 & --- & 0.14 & --- & 0.040558 & 0.039817 \\
--- & 356.9513 & 356.9752 & --- & 0.16 & --- & 0.045994 & 0.045385 \\
--- & 357.3737 & 357.4062 & --- & 0.18 & --- & 0.051728 & 0.051279 \\
--- & 357.8172 & 357.8559 & --- & 0.2 & --- & 0.057838 & 0.05756 \\
358.27 & 358.2827 & 358.3247 & 0.23394 & 0.22 & 0.07235 & 0.064382 & 0.06427
\end{tabular}




\begin{tabular}{|c|c|c|c|c|c|c|c|}
\hline-- & 358.7703 & 358.8123 & --- & 0.24 & --- & 0.071403 & 0.071442 \\
\hline- & 359.2792 & 359.3182 & --- & 0.26 & --- & 0.078935 & 0.079111 \\
\hline & 359.809 & 359.8419 & --- & 0.28 & --- & 0.087022 & 0.087305 \\
\hline & 360.3579 & 360.3829 & - & 0.3 & -- & 0.095651 & 0.096046 \\
\hline 360.69 & 360.9266 & 360.9407 & 0.31309 & 0.32 & 0.10668 & 0.104923 & 0.105359 \\
\hline & 361.5132 & 361.5147 & -- & 0.34 & --- & 0.114797 & 0.11526 \\
\hline & 362.1171 & 362.1048 & 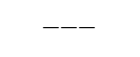 & 0.36 & --- & 0.125306 & 0.125765 \\
\hline-- & 362.7377 & 362.7107 & --- & 0.38 & --- & 0.136467 & 0.136894 \\
\hline 363.36 & 363.3746 & 363.3326 & 0.40133 & 0.4 & 0.15202 & 0.148296 & 0.148665 \\
\hline & 364.0277 & 363.9705 & 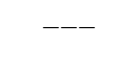 & 0.42 & --- & 0.160812 & 0.161098 \\
\hline & 364.6969 & 364.6248 & 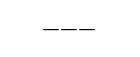 & 0.44 & --- & 0.174034 & 0.174216 \\
\hline 365.28 & 365.3825 & 365.2963 & 0.46408 & 0.46 & 0.19987 & 0.187983 & 0.188045 \\
\hline . & 366.0847 & 365.9855 & - & 0.48 & --- & 0.202684 & 0.202614 \\
\hline & 366.8042 & 366.6935 & --- & 0.5 & --- & 0.218166 & 0.217956 \\
\hline 367.69 & 367.5418 & 367.4215 & 0.51408 & 0.52 & 0.24789 & 0.234462 & 0.234109 \\
\hline--- & 368.2984 & 368.1708 & - & 0.54 & --- & 0.25161 & 0.251118 \\
\hline & 369.0752 & 368.9429 & & 0.56 & & 0.269653 & 0.269034 \\
\hline & 369.8735 & 369.7396 & & 0.58 & & 0.288644 & 0.287917 \\
\hline & 370.6948 & 370.5628 & -- & 0.6 & --- & 0.308639 & 0.307834 \\
\hline & 371.5405 & 371.4147 & & 0.62 & & 0.329705 & 0.328863 \\
\hline & 372.4124 & 372.2974 & --- & 0.64 & 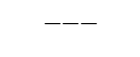 & 0.351918 & 0.351095 \\
\hline 373.28 & 373.3122 & 373.2132 & 0.64749 & 0.66 & 0.36875 & 0.375361 & 0.374629 \\
\hline & 374.2415 & 374.1645 & & 0.68 & --- & 0.400128 & 0.399578 \\
\hline & 375.2018 & 375.1533 & . & 0.7 & 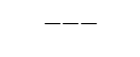 & 0.426319 & 0.426064 \\
\hline 376.39 & 376.1942 & 376.1815 & 0.71275 & 0.72 & 0.45048 & 0.45404 & 0.454217 \\
\hline 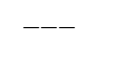 & 377.2195 & 377.2499 & . & 0.74 & --- & 0.483398 & 0.484169 \\
\hline & 378.2774 & 378.3584 & --- & 0.76 & --- & 0.514497 & 0.516043 \\
\hline 379.3 & 379.3666 & 379.5047 & 0.78331 & 0.78 & 0.55121 & 0.547427 & 0.549942 \\
\hline & 380.4841 & 380.6841 & --- & 0.8 & --- & 0.582249 & 0.585918 \\
\hline & 381.6247 & 381.8879 & . & 0.82 & --- & 0.618982 & 0.623951 \\
\hline & 382.7807 & 383.1034 & --- & 0.84 & --- & 0.657578 & 0.663908 \\
\hline & 383.9417 & 384.3175 & -2 & 0.86 & --- & 0.697908 & 0.705554 \\
\hline & 385.0946 & 385.5009 & - & 0.88 & --- & 0.739742 & 0.748398 \\
\hline & 386.2246 & 386.6355 & --- & 0.9 & --- & 0.78275 & 0.791921 \\
\hline & 387.3161 & 387.701 & --- & 0.92 & --- & 0.826517 & 0.835508 \\
\hline & 388.3549 & 388.6798 & --- & 0.94 & --- & 0.870576 & 0.878542 \\
\hline & 389.3289 & 389.5673 & --- & 0.96 & --- & 0.914455 & 0.920552 \\
\hline & 390.2299 & 390.3578 & --- & 0.98 & --- & 0.957718 & 0.961121 \\
\hline 1.09 & 391.054 & 391.0541 & 1 & 1 & 1 & 1 & 1 \\
\hline
\end{tabular}

\section{Identification of the parameters of the reactive distillation column}

Using the EQ-EQ modeling approach, a reactive distillation column was modeled in the present study. The kinetic model obtained from the stationary solution 
in a batch reactor was used to describe the chemical equilibrium of the reactive distillation column. Figure S1, S2, S3, and S4 clearly illustrate the optimisation of theoretical plates number, reflux ratio, HAc feeding position, and MA feeding position.

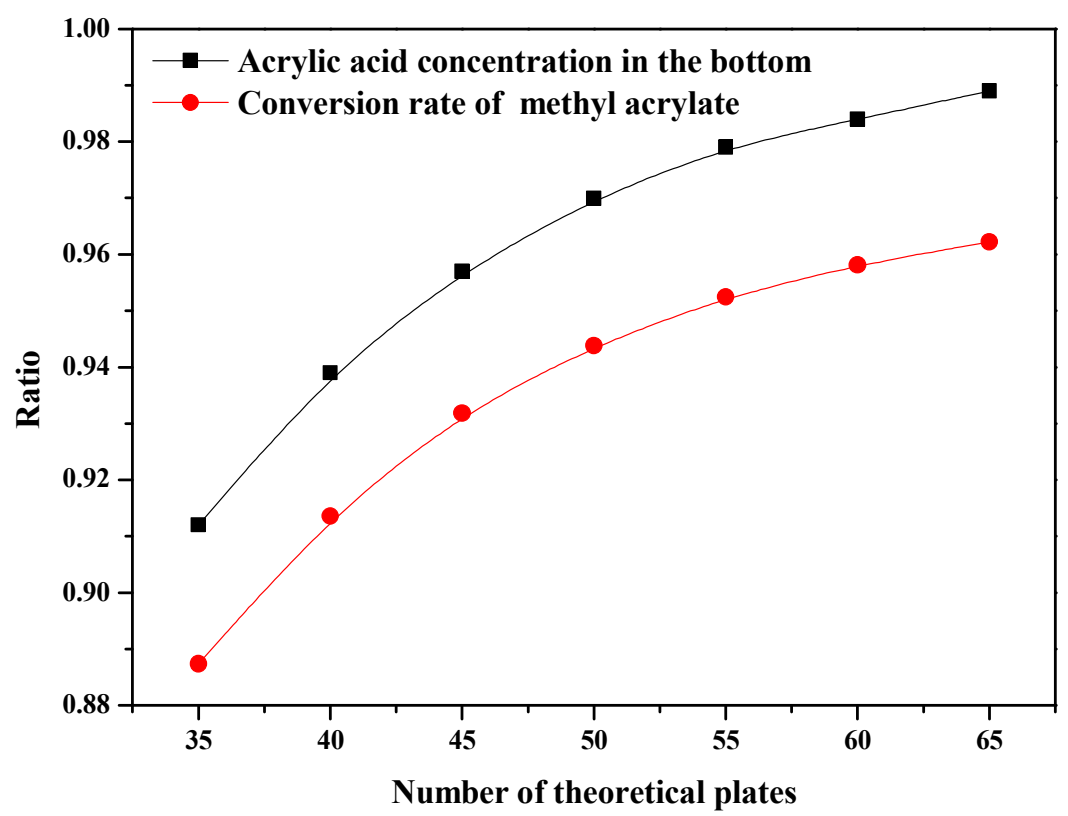

Figure S1. Effect of theoretical plates number on the performance of the column.

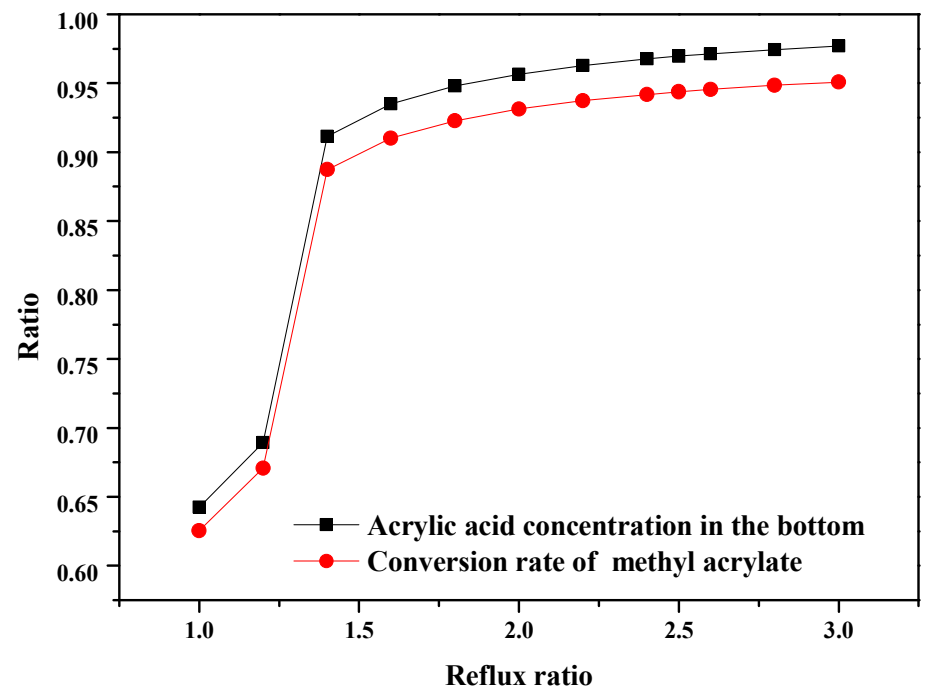

Figure S2. Effect of Reflux ratio on the performance of the column. 


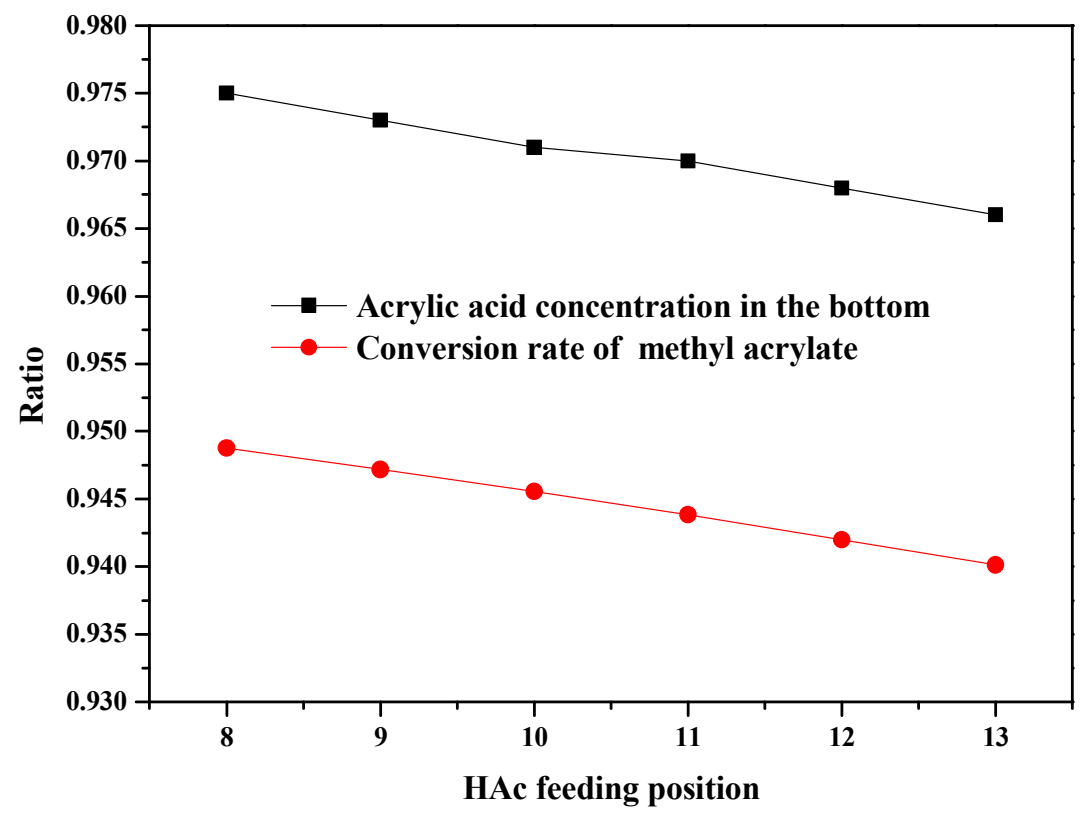

Figure S3. Effect of HAc feeding position on the performance of the column.

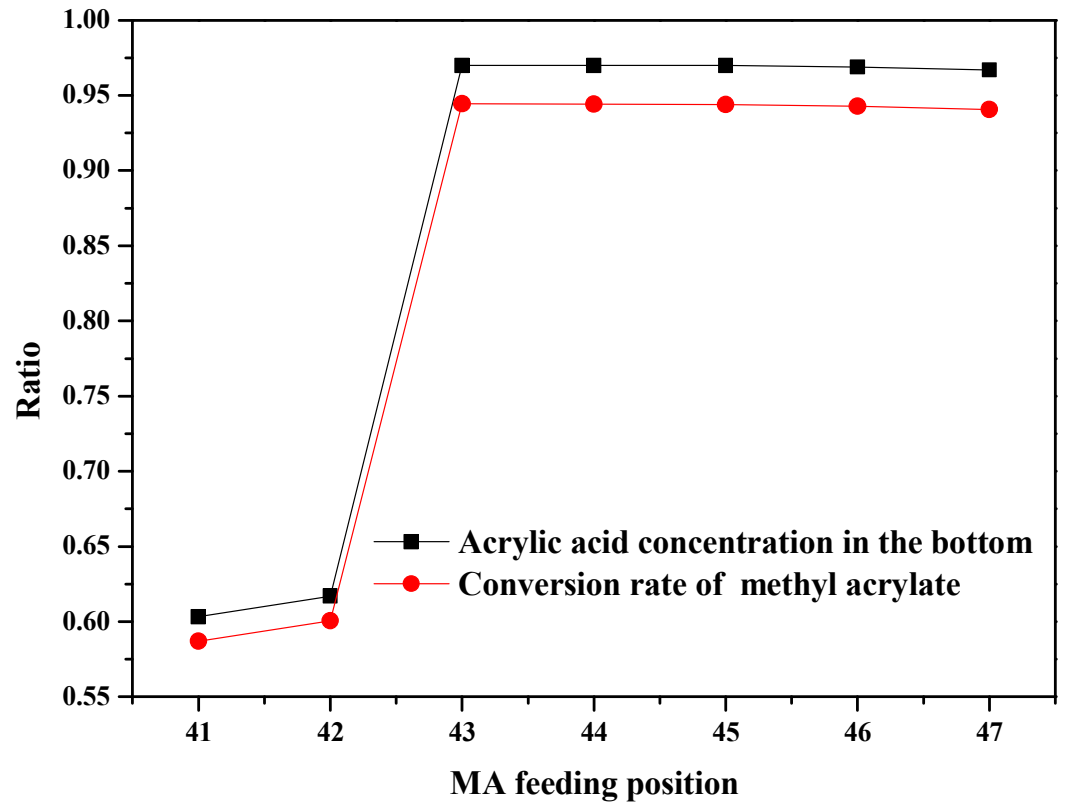

Figure S4. Effect of MA feeding position on the performance of the column. 